\title{
AICAR activates AMPK to regulate STAT3 nuclear translocation and phosphorylation and iNOS expression in inflammatory pain
}

\author{
Hongchun Xiang
}

Department of Neurobiology, School of Basic medicine, Tongji Medical College, Huazhong University of

Science and Technology

\section{Guo-Wei Cai}

Department of Acupuncture-moxibustion, Union Hospital, Huazhong University of Science and

Technology

\section{Liang Hu}

Department of Pharmacology, School of Basic Medicine, Nanjing Medical University

\section{He Zhu}

Department of Neurobiology, School of Basic Medicine, Tongji Medical College, Huazhong University of

Science and Technology

\section{Lixue Lin}

Department of Neurobiology, School of Basic Medicine, Tongji Medical College, Huazhong University of Science and Technology

\section{Yongmin Liu}

Department of Neurobiology, School of Basic Medicine, Tongji Medical College, Huazhong University of Science and Technology

\section{Xuefei Hu}

Department of Neurobiology, School of Basic Medicine, Tongji Medical College, Huazhong University of

Science and Technology

\section{Chao Chen}

Department of Neurobiology, School of Basic Medicine, Tongji Medical College, Huazhong University of Science and Technology

\section{Xiaocui Yuan}

Department of Physiology and Pathophysiology, School of Basic Medical Sciences, Xi'an Jiaotong University

\section{Tao Weng}

Department of Neurobiology, School of Basic Medicine, Tongji Medical College, Huazhong University of Science and Technology

\section{Yan Zou}


Department of Neurobiology, School of Basic Medicine, Tongji Medical College, Huazhong University of Science and Technology

\section{Huilin Pan}

Department of Anethesiology and Pain Medicine, The University of Texas MD Anderson Cancer Center Wentao Liu

Department of Pharmacology, School of Basic Medicine, Nanjing Medical University

\section{Man Li ( $\boldsymbol{\nabla}$ liman73@mails.tjmu.edu.cn )}

Huazhong University of Science and Technology https://orcid.org/0000-0003-4041-0437

\section{Research}

Keywords: AICAR, AMPK, STAT3 phosphorylation, iNOS, inflammatory pain

Posted Date: October 19th, 2020

DOl: https://doi.org/10.21203/rs.3.rs-93480/v1

License: (c) (1) This work is licensed under a Creative Commons Attribution 4.0 International License. Read Full License 


\section{Abstract}

Background: AMP-activated protein kinase (AMPK) activators can improve inflammatory pain and neuropathic pain. Inflammation translocate signal transducers and activators of transcription 3 (STAT3) to the nuclei of activated macrophages, and STAT3 phosphorylation promotes the expression of inducible nitric oxide synthetase (iNOS). In this study, we determined whether AMPK activation alleviate inflammatory pain via STAT3 nuclear translocation and phosphorylation.

Methods: Immunoblotting was used to measure the expression of p-AMPK, and iNOS. Immunoblotting and immunofluorescence were used to detect the nuclear translocation of p-STAT3(Ser727) and STAT3 in macrophages of local inflammatory tissues. Flow cytometry was used to measure reactive oxygen species (ROS) accumulation and mitochondrial damage.

Results: AMPK activation with AICAR significantly alleviated pain hypersensitivity and inhibited the expression of iNOS in complete Freund's adjust (CFA)-induced inflamed skin tissues. CFA caused nuclear translocation of STAT3 and p-STAT3(Ser727) in macrophages of inflamed skin tissues. AICAR inhibited nuclear translocation of STAT3 and p-STAT3(Ser727) and promoted the phosphorylation of STAT3(Ser727) in the cytoplasm of macrophages. AICAR also inhibited the expression of iNOS and nuclear translocation of STAT3 and p-STAT3(Ser727), and promoted the phosphorylation of STAT3(Ser727) in NR8383 macrophages treated with CFA. AMPK activation also inhibited the ROS generation and the mitochondrial damage of NR8383 macrophages caused by CFA. In addition, transfection of STAT3 S727D decreased ROS and alleviated mitochondrial damage.

Conclusions: Activation of AMPK attenuates inflammatory pain and suppresses STAT3 nuclear translocation and phosphorylation of STAT3(Ser727) in macrophages, resulting in reduced iNOS. Activation of AMPK also promotes phosphorylation of STAT3(Ser727) in the cytoplasm of macrophages to alleviate ROS accumulation and mitochondrial damage associated with inflammation.

\section{Background}

Inflammatory pain is a common symptom associated with many clinical conditions[1]. Tissues damage leads to the release of inflammatory mediators from damaged cells[2]. A variety of inflammatory mediators in the extracellular environment causes the sensitization of nociceptive neurons[3]. In formalininduced inflammatory pain model, inhibition of interleukin-1 $\beta$ (IL-1 $\beta$ ), tumor necrosis factor- $\alpha$ (TNF- $\alpha$ ) and inducible nitric oxide synthetase (iNOS) and other inflammatory mediators alleviates inflammatory pain[4]. In addition, activated macrophages release nitric oxide and reactive oxygen species (ROS), which play an important role in inflammation and pain $[5,6]$.

AMP-activated protein kinase (AMPK) is a kinase that regulates energy homeostasis. Phosphorylation of Thr172 site is a marker of AMPK activation[7]. In chronic pain induced by spared nerve injury, activation of AMPK reduces the excitability of dorsal root ganglion neurons to relieve pain[8]. In a mouse model of acute incision-induced pain, local administration of resveratrol cream or systemic administration of 
metformin activates AMPK to alleviate pain[9]. In lipoteichoic acid (LTA)-induced inflammation, the AMPK activator 5-amino-1 - $\beta$-d-ribofuranosyl-imidazole-4-carboxamide (AICAR) inhibits LTA-induced neutrophil influx and cytokines levels in the bronchoalveolar space to alleviate inflammation[10].

iNOS is an important inflammatory factor produced by macrophages[11]. In lipolysaccharide (LPS)induced macrophages, nuclear translocation of STAT3 promotes the expression of iNOS[12].

Phosphorylated STAT3 (p-STAT3, Ser727) in the nuclei increases the expression of iNOS[12, 13]. The stimulation of pro-inflammatory cytokines, such as TNF-a and IL-1 $\beta$, leads to the production of ROS in the mitochondria[14, 15]. Excessive ROS activation of MPTP(mitochondrial permeability transition pore) results in mitochondrial dysfunction[16]. In complete Freund's adjust (CFA)-induced inflammatory pain, cytokines released from activated macrophages play an important role in mediating the peripheral inflammatory response and sensitization of peripheral sensory nerves $[17,18]$. In the model of cerebral ischemia injury, electroacupuncture treatment promotes the phosphorylation of STAT3 (Ser727) significantly, and participates in the protective effect of electroacupuncture on cerebral ischemia injury[19]. In the model of myocardial ischemia-reperfusion injury of rats, $\mathrm{ZnCl}_{2}$ promotes the phosphorylation of STAT3(Ser727) in cytoplasm, and attenuates mitochondrial damage and accumulation of mitochondrial ROS (reactive oxygen species)[20].

It is unclear whether STAT3 phosphorylation in activated macrophages play a role in the analgesic effect of AMPK activation on inflammatory pain. In this study, we determined whether AMPK activation reduces inflammatory pain by regulating STAT3 nuclear translocation and phosphorylation. Our study provides new information about the signaling mechanisms involved in the analgesic effect of AMPK activation on inflammatory pain.

\section{Methods}

\section{Mouse models}

The experimental procedures were approved by the ethics committee of Tongji Medical College of Huazhong University of Science and Technology and carried out in strict accordance with the ethical guidelines of the International Association for the Study of Pain. Male C57BL/6 mice (8-9-week old) were purchased from Beijing Vital River Laboratory Animal Technology Co., Ltd. Four to six mice were housed in each cage ( $12 \mathrm{~h}$-light and $12 \mathrm{~h}$-dark, ambient temperature of $\left.22-24^{\circ} \mathrm{C}\right)$ and had free access to drinking and food. Before experiments, all mice were adapted to the environment. As reported previously[3], $25 \mu \mathrm{l}$ of CFA (Sigma, F5881-10 ml) was subcutaneously injected into the plantar side of the left hindpaw to induce inflammatory pain. The control group received an injection of $25 \mu \mathrm{l}$ normal saline (N.S.).

\section{Nociceptive Behavioral Tests}

The withdrawal threshold of pain was measured three days before injection of CFA or vehicle. After 30 minutes of acclimation, we applied von Frey filaments (Stoelting, wood Dale, Italy) to the plantar surface of the left hindpaw vertically. Each filament was bend for $5 \mathrm{~s}$ with enough force, and the rapid withdrawal 
of the hindpaw was considered a positive reaction. The "up-down" method was used to determine the mechanical pain threshold of mice[21]. Each test was repeated twice, and the average value was used.

Hot-plate was used to measure the thermal pain threshold, and the surface temperature was maintained at $53^{\circ} \mathrm{C}$. When the mice were placed on the hotplate, the latency of rapid paw withdrawal was recorded. The mice were removed immediately after the withdrawal response was observed. We set 20 seconds as the cut-off to prevent tissue damage[22]. The hot-plate test was repeated every 5 minutes for three times, and the averaged value was calculated.

\section{Western Blotting}

Western blot analysis was used to assess the proteins of cytoplasm and nuclear extracts in local inflammatory tissues and NR8383 cells. We used Nucleoprotein and Cytoplasmic Protein Extraction Kit (\#P0027, Beyotime Biotechnology, China) to extract nuclear and cytoplasmic proteins. Mice were anesthetized with $5 \%$ chloralic hydras $(0.33 \mathrm{ml}$ per $10 \mathrm{~g}$ body weight), and local inflammatory skin tissues were removed immediately and minced with scissors. After the treatment of drugs and CFA, the NR8383 cells were centrifuged at $1000 \mathrm{rpm}$ for 5 minutes to collect the sediments. The skin tissues or NR8383 cells were then lysed by adding RIPA Lysis Buffer $(10 \mu \mathrm{l} / \mathrm{mg}$ for tissues, $100 \mu \mathrm{l}$ for cells in a well of sixwell plates, \#P0013B, Beyotime Biotechnology, China) with $1 \mathrm{mM}$ of a protease and phosphatase inhibitor cocktail (Servicebio Biotech, Wuhan, China). The lysate was centrifuged with $12000 \mathrm{~g}\left(4^{\circ} \mathrm{C}\right.$, $15 \mathrm{~min}$ ), and the supernatant was collected. The protein contents of supernatant was detected using BCA kit (\#P0012, Beyotime Biotechnology, China). Protein solution were boiled for $5 \mathrm{~min}$ at $95^{\circ} \mathrm{C}$ with $1 / 4$ volume $5 \times$ loading buffer (Servicebio Biotech, Wuhan, China) and added to SDS-PAGE. Then, we transferred the proteins to the PVDF membrane (Millipore Corp.). The membranes were incubated with $5 \%$ skim milk or $5 \%$ BSA for 1 hour at room temperature. The membrane was probed with the following primary antibodies: p-AMPK (Thr172) (1:1000; Cell Signaling Technology, \#2535), AMPK (1:1000, Cell Signaling Technology, \#2532), p-STAT3 (Ser727) (1:1000, Abcam, \#ab30647), STAT3 (1:1000, Abcam, \#68153); iNOS (1:1000, Abcam, \#178945); Histone-3 (1:10000, Proteintech, \#Cat.No.10265-1-AP); GAPDH (1:8000, Proteintech, \#Cat.No. 60004-1-Ig); $\beta$-actin (1:10000, Santa Cruz Biotechnology, sc-47778). Secondary antibodies were anti-rabbit HRP and Anti-mouse HRP (1:20000).

\section{Quantitative Real-time PCR}

Total RNA was isolated from local inflammatory skin tissues and NR8383 cells using Trizol Reagent (Invitrogen, TRIzol® Reagent,\#15596-018). Spectrophotometer (Thermo Scientific, USA) was used to quantify the concentration of the total RNA. We used Hifair®凶1 st Strand cDNA Synthesis SuperMix for qPCR(gDNA digester plus)to reverse total RNA into cDNA. We used Cham QTM Universal SYBR® qPCR Master Mix (Nanjing, China) on the Applied biosystems QuantStudio 7 FlexQThermo Fisher) for qPCR. Expression values of the iNOS mRNA were normalized to the corresponding expression of $\beta$-actin mRNA. We used 2- $\Delta \Delta$ Ct method to calculate relative expression levels of iNOS mRNA. The sequence-specific primers used are listed below. 
Mouse iNOS: FORWARD, 5'-3' CGGACGAGACGGATAGGCAGAG; REVERSE 5'-3' GGAAGGCAGCGGGCACATG.

Mouse $\beta$-actin: FORWARD, 5'-3' GTGCTATGTTGCTCTAGACTTCG; REVERSE, 5'-3' ATGCCACAGGATTCCATACC.

\section{Immunofluorescence Labeling}

Mice were anesthetized with $10 \%$ chloralic hydras and were transcardially perfused with $37^{\circ} \mathrm{C}$ N.S. followed by $4 \%$ paraformaldehyde in $0.1 \mathrm{M}$ phosphate buffer $\left(\mathrm{pH}, 7.4 ; 4{ }^{\circ} \mathrm{C}\right)$. The local inflammatory skin tissues were quickly removed and post-fixed for $8 \mathrm{~h}$ in the same fixative solution and cryoprotected in $20 \%$ and $30 \%$ sucrose in $0.1 \mathrm{M}$ phosphate buffer for $24 \mathrm{~h}$ at $4{ }^{\circ} \mathrm{C}$. The slices $(15 \mu \mathrm{m}$ in sickness) were cut on a cryostat $\left(-20^{\circ} \mathrm{C}\right)$ and were mounted on a gelatin-coated slide and air dried 6 hours.

For immunofluorescence labeling of cultured cells, the cells were seeded into a 24-well plate with polylysine $(0.01 \%)$-coated slides and then treated with drugs. After experiment, cells were fixed with $4 \%$ paraformaldehyde for 15 minutes and washed with $0.01 \mathrm{M}$ PBS. The sections were washed in $0.01 \mathrm{M}$ PBS and blocked for $1 \mathrm{~h}$ with $5 \%$ donkey serum(0.2\% tween-20)in $0.01 \mathrm{M}$ PBS and then incubated with primary antibodies at $4{ }^{\circ} \mathrm{C}$ overnight. The primary antibodies used are rabbit anti-p-AMPK (Abcam, \#ab23875) (1:250), mouse anti-CD68 (Abcam, \#ab955) (1:200), rabbit anti-p-STAT3ロSer727区(1:300, Abcam, \#ab30647)and rabbit anti-STAT3 (1:300, Abcam, \#68153). The secondary antibodies are donkey antirabbit IgG conjugated with Dylight 594 (1:600) and donkey anti-mouse IgG conjugated with Dylight488 (Jackson Immuno Research, USA). Sections were incubated with DAPI for the nucleus staining for 5 min and then washed 3 times in $0.01 \mathrm{M}$ PBS for $5 \mathrm{~min}$. Sections were cover-slipped with anti-quenching mounting agent. Images were acquired using fluorescence microscope (BX51, Olympus, Japan) and were analyzed using $\mathrm{NIH}$ Image $\mathrm{J}$ software (Bethesda, MD, USA).

\section{Cell Culture And Treatment}

A macrophage cell line, NR8383, was purchased from Procell (Procell, Wuhan, China) and cultured in HyCloneTM DMEM high glucose medium (GE healthcare life sciences, HyClone Laboratories, USA) with penicillin/streptomycin (100 U/10 mg/mL, \#C0222, Beyotime Biotechnology, China) and 20\% (v/v) fetal calf serum (Biological Industries, Kibbutz Beit, Israel). Cells were grown in 50-ml flasks under standard cell culture conditions $\left(37^{\circ} \mathrm{C}, 5 \% \mathrm{CO} 2\right)$.

To determine whether AICAR induces activation of AMPK to decrease iNOS expression, NR8383 cells were treated with AICAR $(0.5 \mathrm{mM})$ and Compound C $(20 \mu \mathrm{M})$ before treatment with N.S. or CFA $(100 \mu \mathrm{g} / \mathrm{ml})$. NR8383 cells were exposed to CFA for 24 hours. We then collected cells for western blotting and flow cytometry.

\section{Lentivirus Transfection}


The control vector (WT) and vectors encoding the STAT3 Ser727 mutant were transfected into NR8383 cells according to the manufacturer's instruction. Briefly, cultured NR8383 cells were transfected for $48 \mathrm{~h}$ with the expression vectors for STAT3 S727D (Lentivirus vector encoding an m-Cherry-STAT3 fusion protein that carries a serine-to-aspartate substitution at codon 727, m-Cherry-STAT3 S727D) and WT STAT3 (Lentivirus vector encoding an m-Cherry-STAT3 fusion protein). The expression of m-CherrySTAT3 and m-Cherry-STAT3 S727D was validated via a fluorescence microscope. Both vectors were constructed by Shanghai Genechem Technology Co., Ltd, China. The transfection efficiency $(70-80 \%)$ was assessed by the percentage of cells with m-Cherry at 48 hours after transfection. The cells were collected for immunofluorescence and flow cytometry at the end of treatments.

\section{Measurement Of Intracellular ROS}

We used 2',7'-dichlorofluorescein diacetate (DCF-DA)(\#S0033, Beyotime Biotechnology, China) to measure intracellular ROS. NR8383 cells were loaded with DCF-DA $(10 \mu \mathrm{M})$ diluted with serum-free medium and incubated for 30 minutes at $37^{\circ} \mathrm{C}$. Then, cells were washed and resuspended with $0.01 \mathrm{M}$ PBS. Finally, we used BD LSRFortessaTM, Special Order Research Product (FITC channel) to detect the fluorescence intensity of DCF. Flow Jo 7.6 was used to analyze data.

\section{Measurement Of Mitochondrial Dysfunction}

NR8383 cells were loaded with Mito-Tracker Red CMXRos (200 nM, \#C1049, Beyotime Biotechnology, China). CMXRos can be used to label bioactivaty mitochondria of living cells and display red fluorescence. We use the drug strictly according to the instructions. Mito-Tracker Red CMXRos was diluted with complete medium and incubated for 30 minutes at $37^{\circ} \mathrm{C}$. Then, cells were washed and resuspended with $0.01 \mathrm{M}$ PBS. Finally, we used BD LSRFortessa ${ }^{\mathrm{TM}}$ (PE-Texas Red-A channel) to detect the fluorescence intensity of. Flow Jo 7.6 was used to analyze data.

\section{Statistical analysis}

All data were expressed by mean \pm SEM. The two-way analysis of variance (ANOVA) followed by Bonferroni's post hoc test were used to determine statistical difference in the withdrawal thresholds in different groups (SPSS, version 11.0). One-way ANOVA and Newman Keuls post hoc test were used to analyze the expression level of protein and fluorescence intensity. The comparison between the two groups was made using non-paired Student's t test. $p<0.05$ was considered statistically significant.

\section{Results}

\section{AICAR reduces CFA-induced pain hypersensitivity and increases AMPK phosphorylation}

Mice received CFA injection exhibited mechanical allodynia and thermal hyperalgesia (Fig. 1). A single subcutaneous administration of AICAR $(20 \mu \mathrm{g})$ at day 7 after CFA injection suppressed mechanical allodynia and thermal hyperalgesia significantly (Fig. 1a, b). AICAR also increased the phosphorylation of AMPK (Fig. 1c-e). p-AMPK was present in CD68-positive macrophages of inflammatory skin tissues 
(Fig. 1f). The results suggest that activation of AMPK with AICAR in inflammatory skin tissues reduces inflammatory pain.

\section{Activation of AMPK with AICAR inhibits the expression of iNOS}

Immunoblotting and qPCR were used to test the effect of AMPK activation on iNOS expression in inflamed skin tissues. CFA treatment significantly increased in the protein and mRNA levels of iNOS (Fig. $1 \mathrm{~g}, \mathrm{~h}$ and i). Treatment with AICAR via a single subcutaneous injection at day 7 after CFA injection significantly inhibited the expression levels of iNOS (Fig. 1g, h and i).

\section{Activation of AMPK inhibits STAT3 nuclear translocation and promotes cytoplasmic STAT3 (Ser727) phosphorylation in macrophages}

We next determined whether activation of AMPK affects STAT3 nuclear translocation and phosphorylation in macrophages. CFA treatment significantly increased the protein level of p-STAT3 (Ser727), and AICAR further increased p-STAT3 (ser727) significantly in inflammatory skin tissues (Fig. 2a, b). There was no significant difference in the total protein level of STAT3 between the control, CFA, and CFA plus AICAR groups (Fig. 2a, c).

In addition, we tested the protein levels of STAT3 and p-STAT3 (Ser727) in the cytoplasmic and nuclear fractions of inflamed skin tissues. CFA significantly increased the protein level of p-STAT3 (Ser727) and STAT3 in nuclear fraction of inflammatory skin tissues. AICAR reversed this effect of CFA (Fig. 2d-f). Compared with the CFA model group, AICAR significantly increased the protein level of p-STAT3(Ser727) in the cytoplasmic fraction of inflammatory skin tissues (Fig. 2g-i). Immunofluorescence labeling showed the expression of STAT3 and p-STAT3 (Ser727) in macrophages of inflammatory skin tissues(Fig. 2j, k). These results suggest that CFA induces the nuclear translocation of STAT3 and increases STAT3(Ser727) phosphorylation in macrophages of local inflammatory skin tissues. Also, AMPK activation inhibits nuclear translocation of STAT3 and increases the phosphorylation of STAT3(Ser727) in the cytoplasm of macrophages.

\section{The AMPK activation in macrophages promotes the phosphorylation of STAT3 (Ser727) in cytoplasm and inhibit the nuclear translocation of STAT3 and the expression of iNOS}

NR8383 cells were used to induce macrophage inflammation with CFA treatment[19]. CFA treatment significantly increased iNOS protein levels in NR8383 cells(Fig. 3). Compared with the CFA group, AICAR significantly inhibited the expression of iNOS and increased the phosphorylation of STAT3 (Ser727). AICAR had no significant effect on total protein levels of STAT3. Compound C, an AMPK antagonist, reversed the effect of AICAR (Fig. 3a-d). These data suggest that activation of AMPK enhances the phosphorylation of STAT3 (Ser727) and inhibits the expression of iNOS in activated macrophages.

Furthermore, in NR8383 cells, we determined whether AMPK activation inhibits STAT3 nuclear translocation. Compared with the control group, CFA treatment significantly increased the levels of $p$ STAT3 (Ser727) and STAT3 in the nuclei and significantly decreased the levels of p-STAT3 (Ser727) and 
STAT3 in the cytoplasm. Treatment with AICAR reversed the effects of CFA on the levels of p-STAT3 (Ser727) and STAT3 in the nuclei and the levels of p-STAT3 (Ser727) and STAT3 in the cytoplasm (Fig. 4). In addition, the AMPK antagonist Compound $C$ blocked the effect of AICAR in the above experiments (Fig. 4a-e). Immunofluorescence labeling confirmed the effects of CFA and AMPK activation on the distribution of p-STAT3 (Ser727) and STAT3 in the cytoplasm and nuclei of macrophages(Fig. 4f).

\section{Activation of AMPK alleviates ROS accumulation and mitochondrial damage induced by CFA}

$\mathrm{ZnCl}_{2}$ treatment promotes the phosphorylation of STAT3 (Ser727) in the cytoplasm (mitochondria) and attenuates the mitochondrial damage and ROS accumulation to alleviate the inflammatory damage in a rat model of myocardial ischemia-reperfusion injury[20]. We next used NR8383 cells to determine whether AMPK activation reduces ROS accumulation and mitochondrial damage induced by CFA. Compared with the control group, CFA significantly increased the fluorescence intensity of DCF and significantly decreased the fluorescence intensity of Mito tracker red CMXRos. Treatment with AICAR reduced CFAinduced changes in DCF fluorescence intensity and the fluorescence intensity of Mito tracker red CMXRos in NR8383 cells (Fig. 5). Co-treatment with Compound C reversed the effects of AICAR (Fig. 5a-d). These results suggest that inflammation induces ROS accumulation and mitochondrial damage in macrophages and that activation of AMPK attenuates these inflammation-induced effects.

\section{Promoting STAT3(Ser727) phosphorylation alleviates CFA-induced ROS accumulation and mitochondrial damage in macrophages}

The above experiments showed that AMPK activation promotes the phosphorylation of STAT3 (Ser727) in cytoplasm of macrophages. We then determined whether STAT3 (Ser727) phosphorylation plays a role in the inhibitory effect of AMPK activation on CFA-induced ROS accumulation and mitochondrial damage in NR8383 macrophages. The Ser727 site of STAT3 was mutated to aspartate, S727D, to promote phosphorylation of STAT3, as reported previously[15, 23]. We used m-Cherry-STAT3 S727D (and m-Cherry STAT3 wild type(WT) as a negative control) lentiviral vectors to transfect NR8383 macrophages. CFA treatment significantly increased the ROS level in NR8383 cells transfected with STAT3 WT vectors. In NR8383 cells transfected with STAT3 S727D vectors, the CFA-induced ROS level was significantly decreased (Fig. 6a and b).

In addition, we found that the fluorescence intensity of Mito tracker Red CMXRos was significantly more in NR8383 cells transfected with STAT3 S727D vectors than in NR8383 cells transfected with STAT3 WT vectors (Fig. 6c-e). These results suggest that AMPK activation alleviates ROS accumulation and mitochondrial damage in inflammation-induced macrophages through promoting phosphorylation of STAT3(Ser727).

\section{Discussion}

In this study, we used an inflammatory pain model to determine the signaling mechanism involved in the analgesic effect produced by local AMPK activation. AMPK is a member of the family of metabolically 
sensitive protein kinases, which contains a-catalytic subunits and $\beta$-and $y$-regulatory subunits[24, 25]. AMPK activation can inhibit a variety of pro-inflammatory signal cascades, including c-Jun N-terminal kinase (JNK), nuclear factor kappa B (NF kappa B) and JAK-STAT (Janus kinase signal transducer and activator of transcription)[26-29]. When AMPKa is knocked out in mice, the nociceptive response is enhanced in inflammatory pain[30,31]. In the rat model of osteoarthritis, activation of AMPK/mTOR (mammary target of rapamycin) reduces the inflammatory response of chondrocytes induced by IL-1 $\beta$ and promotes the autophagy to reduce inflammatory injury and osteoarthritic pain[32].

In our study, we showed that activation of AMPK in macrophages inhibited the expression of iNOS and reduced inflammatory pain. We also showed that AMPK activation regulates STAT3 to inhibit iNOS expression in inflammatory pain. As an important neurotransmitter, nitric oxide mediates the pain in peripheral injury model[33,34]. The iNOS is increased in inflammatory and neuropathic pain, which promotes the synthesis of nitric oxide to induce the sensitization of peripheral or central sensory nerves and pain[35,36]. Inhibition of iNOS expression in macrophages is involved in alleviating inflammatory pain[37]. In carrageenan induced hindpaw inflammatory pain mice model, angelica dahurica can alleviate inflammatory pain by inhibiting the production of iNOS and nitric oxide in macrophages[38]. In the rat model of temporomandibular joint osteoarthritis, AMPK activation inhibits IL-1 $\beta$ and nitric oxide, and alleviates nociceptive pain[39].

STAT3 is a member of the signal transducer and activator of transcription (STAT) family, which includes seven members, STAT 1, STAT 2, STAT 3, STAT 4, STAT 5A, STAT 5B and STAT 6. STAT3 usually transmits signals from activated receptors or intracellular kinases to the nucleus, thus activating and regulating gene transcription[40]. STAT3 activation is induced by a variety of cytokines and growth factors, including IL-6 signal transduction receptor chain gp130 (such as IL-6, tumor suppressor M, interleukin-11) or homodimeric cytokine receptor (such as granulocyte colony stimulating factor G-CSF), as well as growth factors (such as epidermal growth factor) acting through protein tyrosine kinase receptor[41]. Under the stimulation of cytokines, STAT3 tyrosine phosphorylation has transcription activity, and nuclear translocation of STAT3 dimer achieves the transcription of target genes[42-44]. Phosphorylation of STAT3 (Ser727) enhances transcription activity of STAT3[41]. In Raw 264.7 macrophage cell line, LPS causes nuclear translocation of STAT3, and p-STAT3 (Ser727) promotes the transcription of iNOS in nuclei[12]. In LPS-induced sepsis rat model, the activation of STAT3 by miR-34a mediates the expression and secretion of iNOS in pulmonary macrophages[45]. In addition, $\mathrm{ZnCl}_{2}$ promotes the phosphorylation of STAT3 (Ser727) in the cytoplasm, and improves the mitochondrial damage and accumulation of ROS to alleviate the inflammatory injury[15]. In our study, we found that CFA caused nuclear translocation of STAT3 in activated macrophages, and p-STAT3 (Ser727) in the nuclei promoted the expression of iNOS. AICAR activates AMPK to inhibit nuclear translocation of STAT3 in activated macrophage, and reduces p-STAT3(Ser727) in nuclei to downregulate expression of iNOS.

Reactive oxygen species (ROS) are intermediate products of normal oxygen metabolism, which are involved in the regulation of cell proliferation and inflammatory processes[46]. ROS is also involved in the pain and can recruit CX3CR1-positive monocyte macrophages to the site of peripheral nerve injury after 
cancer chemotherapy, which can release ROS to activate TRPA1 (transient receptor potential ankyrin 1) channels[47]. In this study, we found that AMPK activation promotes the phosphorylation of STAT3 Ser727 in the cytoplasm of macrophages to reduce the ROS accumulation and mitochondrial damage induced by CFA.

\section{Conclusions}

Our study shows new information about the signaling mechanism involved in the antinociceptive effect of activating AMPK on inflammatory pain. Activation of AMPK inhibited the nuclear translocation of STAT3 and decreased the phosphorylation of STAT3(Ser727) in macrophages, thus reducing the expression of iNOS. AMPK activation also promoted cytoplasmic STAT3(Ser727) phosphorylation in macrophages to reduce ROS accumulation and mitochondrial damage (Fig. 7). This signaling cascade likely play a role in the analgesic effect of AMPK activators on inflammatory pain.

\section{Abbreviations}

AMPK: Adenosine monophosphate (AMP)-activated protein kinase

STAT3: Signal Transducers and Activators of Transcription 3

iNOS: inducible nitric oxide synthetase

CFA: Complete Freund's Adjuvant

ROS: reactive oxygen species

AICAR: 5-aminoimidazole-4-carboxyamide ribonucleoside

PCR: polymerase chain reaction

mRNA: messenger Ribonucleic Acid

WB: Western blot

IF: Immunofluorescence

Ser727: Serine 727

\section{Declarations}

\section{Acknowledgments}

Not applicable. 
Not applicable.

\section{Authors' contributions}

Man Li, Guo-Wei Cai and Wen-Tao Liu conceived and designed the experiments. Hong-Chun Xiang did most of the experiments and analyzed the data. He Zhu, Yong-Min Liu and Xue-Fei Hu helped with mice model and behavior test experiments. Tao Weng and Yan Zou helped with the western blotting experiment. Chao Chen and Xiao-Cui Yuan helped with the data collection. Liang Hu and Li-Xue Lin helped with the cell culture and flow cytometric analyses. Hong-Chun Xiang, Man Li and Hui-Lin Pan wrote the manuscript. All authors reviewed the manuscript.

\section{Funding}

This work was supported by the National Natural Science Foundation of China(No. 81973949).

\section{Ethics approval and consent to participate}

The experimental procedures were approved by the ethics committee of Tongji Medical College of Huazhong University of Science and Technology and carried out in strict accordance with the ethical guidelines of the International Association for the Study of Pain.

\section{Consent for publication}

Not applicable.

\section{Competing Interests}

All authors declare that there is no conflict of interest.

\section{References}

1. Scanzello CR. Role of low-grade inflammation in osteoarthritis. Current opinion in rheumatology. 2017; 29: 79-85.

2. Pedersen JL. Inflammatory pain in experimental burns in man. Danish medical bulletin. 2000; 47: 16895.

3. Liao HY, Hsieh CL, Huang CP, Lin YW. Electroacupuncture Attenuates CFA-induced Inflammatory Pain by suppressing Nav1.8 through S100B, TRPV1, Opioid, and Adenosine Pathways in Mice. Scientific reports. 2017; 7: 42531.

4. Vendramini-Costa DB, Spindola HM, de Mello GC, Antunes E, Pilli RA, de Carvalho JE. Anti-inflammatory and antinociceptive effects of racemic goniothalamin, a styryl lactone. Life sciences. 2015; 139: 83-90. 
5. Qin BH, Liu XQ, Yuan QY, Wang J, Han HY. Anti-Inflammatory Triterpenoids from the Caulophyllum robustum Maximin LPS-Stimulated RAW264.7 Cells. Molecules. 2018; 23(5): 1149.

6. Saragusti AC, Bustos PS, Pierosan L, Cabrera JL, Chiabrando GA, Santos AR, et al. Involvement of the L-arginine-nitric oxide pathway in the antinociception caused by fruits of Prosopis strombulifera (Lam.) Benth. Journal of ethnopharmacology. 2012; 140: 117-22.

7. Price TJ, Dussor G. AMPK: An emerging target for modification of injury-induced pain plasticity. Neuroscience letters. 2013; 557 Pt A: 9-18.

8. Melemedjian OK, Asiedu MN, Tillu DV, Sanoja R, Yan J, Lark A, et al. Targeting adenosine monophosphate-activated protein kinase (AMPK) in preclinical models reveals a potential mechanism for the treatment of neuropathic pain. Molecular pain. 2011; 7: 70.

9. Burton MD, Tillu DV, Mazhar K, Mejia GL, Asiedu MN, Inyang K, et al. Pharmacological activation of AMPK inhibits incision-evoked mechanical hypersensitivity and the development of hyperalgesic priming in mice. Neuroscience. 2017; 359: 119-29.

10. Martin LM, Moller M, Weiss U, Russe OQ, Scholich K, Pierre S, et al. 5-Amino-1-beta-D-RibofuranosylImidazole-4-Carboxamide (AICAR) Reduces Peripheral Inflammation by Macrophage Phenotype Shift. International journal of molecular sciences. 2019; 20(13):3255.

11. Cheng Y, Feng Y, Xia Z, Li X, Rong J. omega-Alkynyl arachidonic acid promotes anti-inflammatory macrophage $\mathrm{M} 2$ polarization against acute myocardial infarction via regulating the cross-talk between PKM2, HIF-1alpha and iNOS. Biochimica et biophysica acta Molecular and cell biology of lipids. 2017; 1862: $1595-605$.

12. Park SY, Baik YH, Cho JH, Kim S, Lee KS, Han JS. Inhibition of lipopolysaccharide-induced nitric oxide synthesis by nicotine through S6K1-p42/44 MAPK pathway and STAT3 (Ser 727) phosphorylation in Raw 264.7 cells. Cytokine. 2008; 44: 126-34.

13. Yu Q, Zeng K, Ma X, Song F, Jiang Y, Tu P, et al. Resokaempferol-mediated anti-inflammatory effects on activated macrophages via the inhibition of JAK2/STAT3, NF-kappaB and JNK/p38 MAPK signaling pathways. International immunopharmacology. 2016; 38: 104-14.

14. Lee IT, Yang CM. Role of NADPH oxidase/ROS in pro-inflammatory mediators-induced airway and pulmonary diseases. Biochemical pharmacology. 2012; 84: 581-90.

15. Dan Dunn J, Alvarez LA, Zhang X, Soldati T. Reactive oxygen species and mitochondria: A nexus of cellular homeostasis. Redox biology. 2015; 6: 472-85.

16. Rottenberg $\mathrm{H}$, Hoek JB. The path from mitochondrial ROS to aging runs through the mitochondrial permeability transition pore. Aging cell. 2017; 16: 943-55. 
17. Gao F, Xiang HC, Li HP, Jia M, Pan XL, Pan HL, et al. Electroacupuncture inhibits NLRP3 inflammasome activation through CB2 receptors in inflammatory pain. Brain, behavior, and immunity. 2018; 67: 91-100.

18. Ghasemlou N, Chiu IM, Julien JP, Woolf CJ. CD11b+Ly6G- myeloid cells mediate mechanical inflammatory pain hypersensitivity. Proceedings of the National Academy of Sciences of the United States of America. 2015; 112: E6808-17.

19. Zhou H, Zhang Z, Wei H, Wang F, Guo F, Gao Z, et al. Activation of STAT3 is involved in neuroprotection by electroacupuncture pretreatment via cannabinoid $\mathrm{CB} 1$ receptors in rats. Brain research. 2013; 1529: 154-64.

20. Zhang G, Sheng M, Wang J, Teng T, Sun Y, Yang Q, et al. Zinc improves mitochondrial respiratory function and prevents mitochondrial ROS generation at reperfusion by phosphorylating STAT3 at Ser(727). Journal of molecular and cellular cardiology. 2018; 118: 169-82.

21. Chaplan SR, Bach FW, Pogrel JW, Chung JM, Yaksh TL. Quantitative assessment of tactile allodynia in the rat paw. Journal of neuroscience methods. 1994; 53: 55-63.

22. Chen G, Kim YH, Li H, Luo H, Liu DL, Zhang ZJ, et al. PD-L1 inhibits acute and chronic pain by suppressing nociceptive neuron activity via PD-1. Nature neuroscience. 2017; 20: 917-26.

23. D'Annessa I, Gandaglia A, Brivio E, Stefanelli G, Frasca A, Landsberger N, et al. Tyr120Asp mutation alters domain flexibility and dynamics of MeCP2 DNA binding domain leading to impaired DNA interaction: Atomistic characterization of a Rett syndrome causing mutation. Biochimica et biophysica acta General subjects. 2018; 1862: 1180-9.

24. Lage R, Dieguez C, Vidal-Puig A, Lopez M. AMPK: a metabolic gauge regulating whole-body energy homeostasis. Trends in molecular medicine. 2008; 14: 539-49.

25. Peixoto CA, Oliveira WH, Araujo S, Nunes AKS. AMPK activation: Role in the signaling pathways of neuroinflammation and neurodegeneration. Experimental neurology. 2017; 298: 31-41.

26. Zhang Y, Qiu J, Wang X, Zhang Y, Xia M. AMP-activated protein kinase suppresses endothelial cell inflammation through phosphorylation of transcriptional coactivator p300. Arteriosclerosis, thrombosis, and vascular biology. 2011; 31: 2897-908.

27. He C, Li H, Viollet B, Zou MH, Xie Z. AMPK Suppresses Vascular Inflammation In Vivo by Inhibiting Signal Transducer and Activator of Transcription-1. Diabetes. 2015; 64: 4285-97.

28. Rutherford C, Speirs C, Williams JJ, Ewart MA, Mancini SJ, Hawley SA, et al. Phosphorylation of Janus kinase 1 (JAK1) by AMP-activated protein kinase (AMPK) links energy sensing to antiinflammatory signaling. Science signaling. 2016; 9: ra109. 
29. Mancini SJ, White AD, Bijland S, Rutherford C, Graham D, Richter EA, et al. Activation of AMP-activated protein kinase rapidly suppresses multiple pro-inflammatory pathways in adipocytes including IL-1 receptor-associated kinase-4 phosphorylation. Molecular and cellular endocrinology. 2017; 440: 44-56.

30. Jeon SM. Regulation and function of AMPK in physiology and diseases. Experimental \& molecular medicine. 2016; 48: e245.

31. Russe OQ, Moser CV, Kynast KL, King TS, Stephan H, Geisslinger G, et al. Activation of the AMPactivated protein kinase reduces inflammatory nociception. The journal of pain : official journal of the American Pain Society. 2013; 14: 1330-40.

32. Zhao X, Li Y, Lin X, Wang J, Zhao X, Xie J, et al. Ozone induces autophagy in rat chondrocytes stimulated with IL-1beta through the AMPK/mTOR signaling pathway. Journal of pain research. 2018; 11: 3003-17.

33. Yang Y, Zhang J, Liu Y, Zheng Y, Bo J, Zhou X, et al. Role of nitric oxide synthase in the development of bone cancer pain and effect of L-NMMA. Molecular medicine reports. 2016; 13: 1220-6.

34. Miyamoto T, Dubin AE, Petrus MJ, Patapoutian A. TRPV1 and TRPA1 mediate peripheral nitric oxideinduced nociception in mice. PloS one. 2009; 4: e7596.

35. Tang Q, Svensson Cl, Fitzsimmons B, Webb M, Yaksh TL, Hua XY. Inhibition of spinal constitutive NOS-2 by $1400 \mathrm{~W}$ attenuates tissue injury and inflammation-induced hyperalgesia and spinal p38 activation. The European journal of neuroscience. 2007; 25: 2964-72.

36. De Alba J, Clayton NM, Collins SD, Colthup P, Chessell I, Knowles RG. GW274150, a novel and highly selective inhibitor of the inducible isoform of nitric oxide synthase (iNOS), shows analgesic effects in rat models of inflammatory and neuropathic pain. Pain. 2006; 120: 170-81.

37. Chen LC, Lin YY, Jean YH, Lu Y, Chen WF, Yang SN, et al. Anti-inflammatory and analgesic effects of the marine-derived compound comaparvin isolated from the crinoid Comanthus bennetti. Molecules. 2014; 19: 14667-86.

38. Kang OH, Chae HS, Oh YC, Choi JG, Lee YS, Jang HJ, et al. Anti-nociceptive and anti-inflammatory effects of Angelicae dahuricae radix through inhibition of the expression of inducible nitric oxide synthase and NO production. The American journal of Chinese medicine. 2008; 36: 913-28.

39. Zhang S, Teo KYW, Chuah SJ, Lai RC, Lim SK, Toh WS. MSC exosomes alleviate temporomandibular joint osteoarthritis by attenuating inflammation and restoring matrix homeostasis. Biomaterials. 2019; 200: $35-47$.

40. Kiu H, Nicholson SE. Biology and significance of the JAK/STAT signalling pathways. Growth factors. 2012; 30: 88-106. 
41. Hillmer EJ, Zhang H, Li HS, Watowich SS. STAT3 signaling in immunity. Cytokine \& growth factor reviews. 2016; 31: 1-15.

42. Akira S, Nishio Y, Inoue M, Wang XJ, Wei S, Matsusaka T, et al. Molecular cloning of APRF, a novel IFNstimulated gene factor 3 p91-related transcription factor involved in the gp130-mediated signaling pathway. Cell. 1994; 77: 63-71.

43. Yu CL, Meyer DJ, Campbell GS, Larner AC, Carter-Su C, Schwartz J, et al. Enhanced DNA-binding activity of a Stat3-related protein in cells transformed by the Src oncoprotein. Science. 1995; 269: 81-3.

44. Levy DE, Darnell JE, Jr. Stats: transcriptional control and biological impact. Nature reviews Molecular cell biology. 2002; 3: 651-62.

45. Cheng DL, Fang HX, Liang Y, Zhao Y, Shi CS. MicroRNA-34a promotes iNOS secretion from pulmonary macrophages in septic suckling rats through activating STAT3 pathway. Biomedicine \& pharmacotherapy = Biomedecine \& pharmacotherapie. 2018; 105: 1276-82.

46. Gupta S, Goldberg JM, Aziz N, Goldberg E, Krajcir N, Agarwal A. Pathogenic mechanisms in endometriosis-associated infertility. Fertility and sterility. 2008; 90: 247-57.

47. Malcangio M. Role of the immune system in neuropathic pain. Scandinavian journal of pain. 2019; 20: 33-7.

\section{Figures}



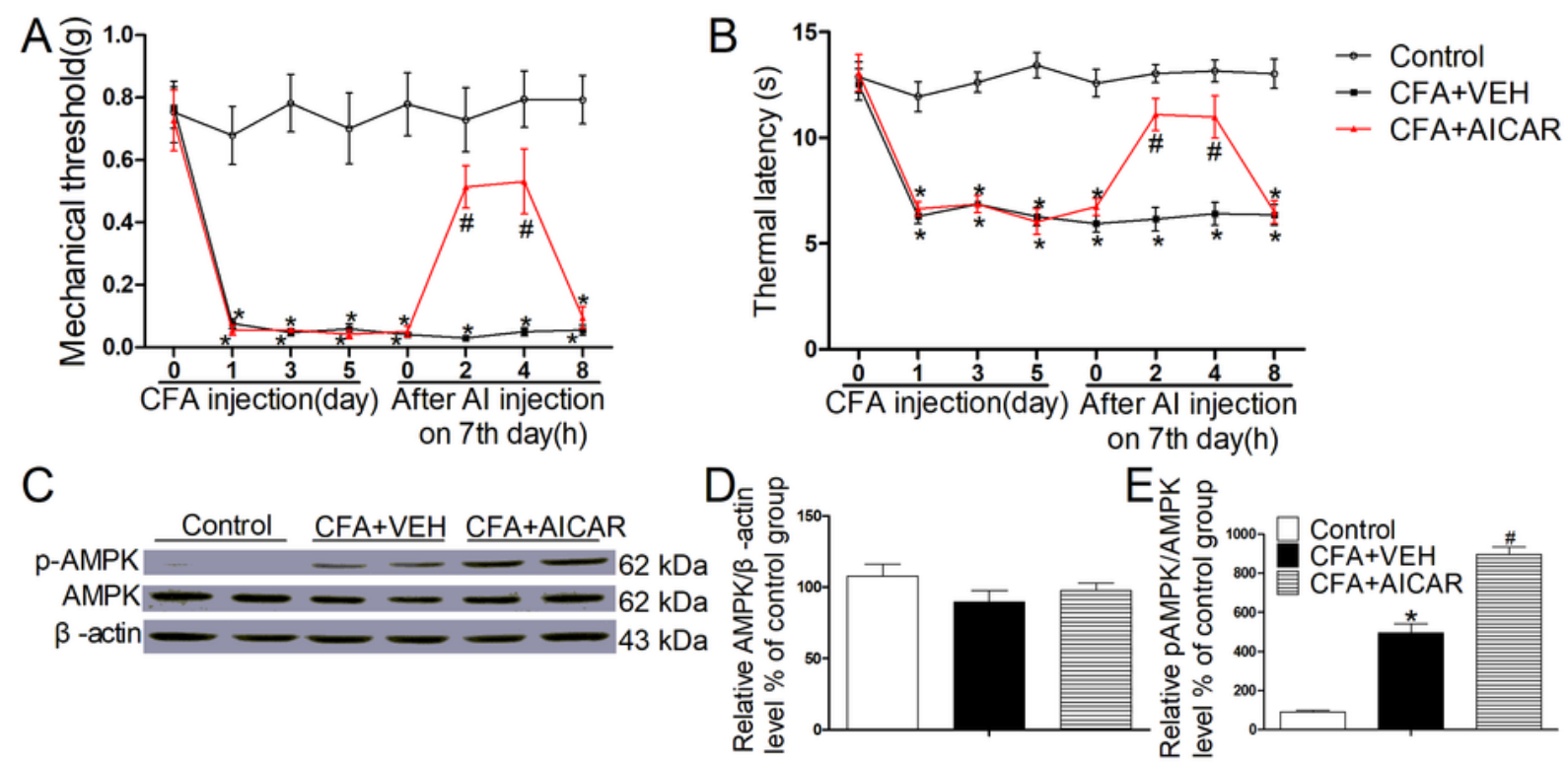

$\mathrm{F}$
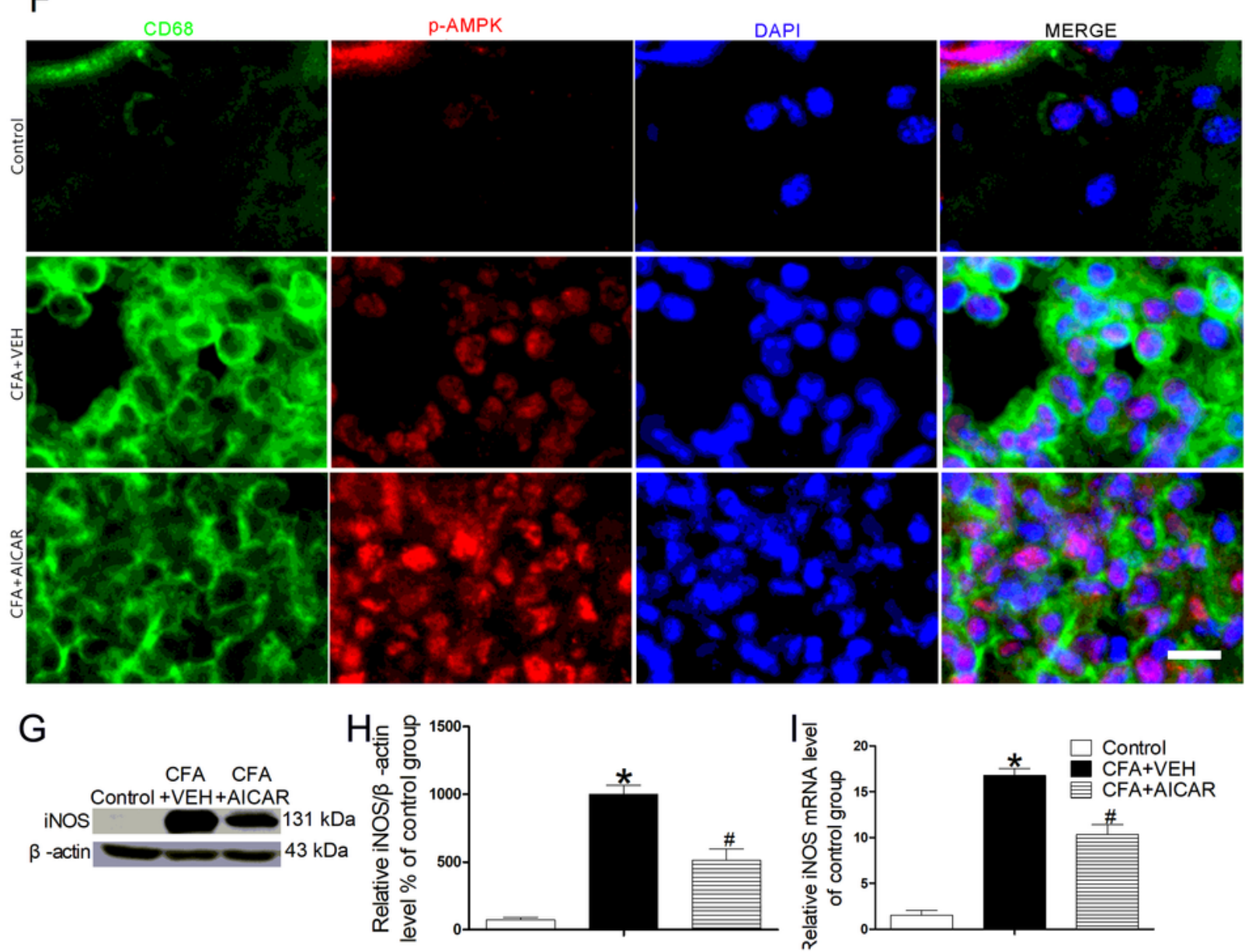

\section{Figure 1}

AICAR reduces inflammatory pain and increases AMPK phosphorylation, and inhibits protein and mRNA levels of iNOS in inflamed skin tissues. (A, B) Mechanical allodynia and thermal hyperalgesia were measured at 2, 4 and 8 hours after treatment with AICAR or vehicle (N.S.) in the 7th day. AICAR $(20 \mu \mathrm{g} / 20 \mu \mathrm{l})$ was injected locally into the inflammatory site at day 7 after CFA injection. ${ }^{*} \mathrm{p}<0.05 \mathrm{vs}$. Control group and $\# p<0.05$ vs. CFA+VEH group ( $n=8$ per group, two-way ANOVA). (C, D and E) Gel 
images and group data showing changes in AMPK/p-AMPK in inflamed skin tissues $(n=3)$. Local inflammatory skin tissues were collected for Western blotting 2 hours after treatment with AICAR or vehicle. ${ }^{*} p<0.05$ vs. Control group and $\# p<0.05$ vs. CFA+VEH group (one-way ANOVA). (F) Immunofluorescence images show co-localization of CD68 (green), p-AMPK (red) and DAPI in inflammatory skin tissues. Scale bar represents $50 \mu \mathrm{m}$. $(\mathrm{G}, \mathrm{H}) \mathrm{Gel}$ images and group data show iNOS proteins in inflamed skin tissues $(n=3)$. AICAR $(20 \mu \mathrm{g} / 20 \mu \mathrm{l})$ was injected locally at day 7 after CFA-induced inflammation. Local inflammatory skin tissues were collected for Western blot and qPCR 2 hours after treatment with AICAR or vehicle. (I) mRNA level of iNOS in inflamed skin tissues $(n=3) .{ }^{*} p<0.05$ vs. Control group and $\# p<0.05$ vs. CFA+VEH group (one-way ANOVA). VEH is the vehicle of $\operatorname{AICAR}(0.01 \mathrm{M}$ PBS). 

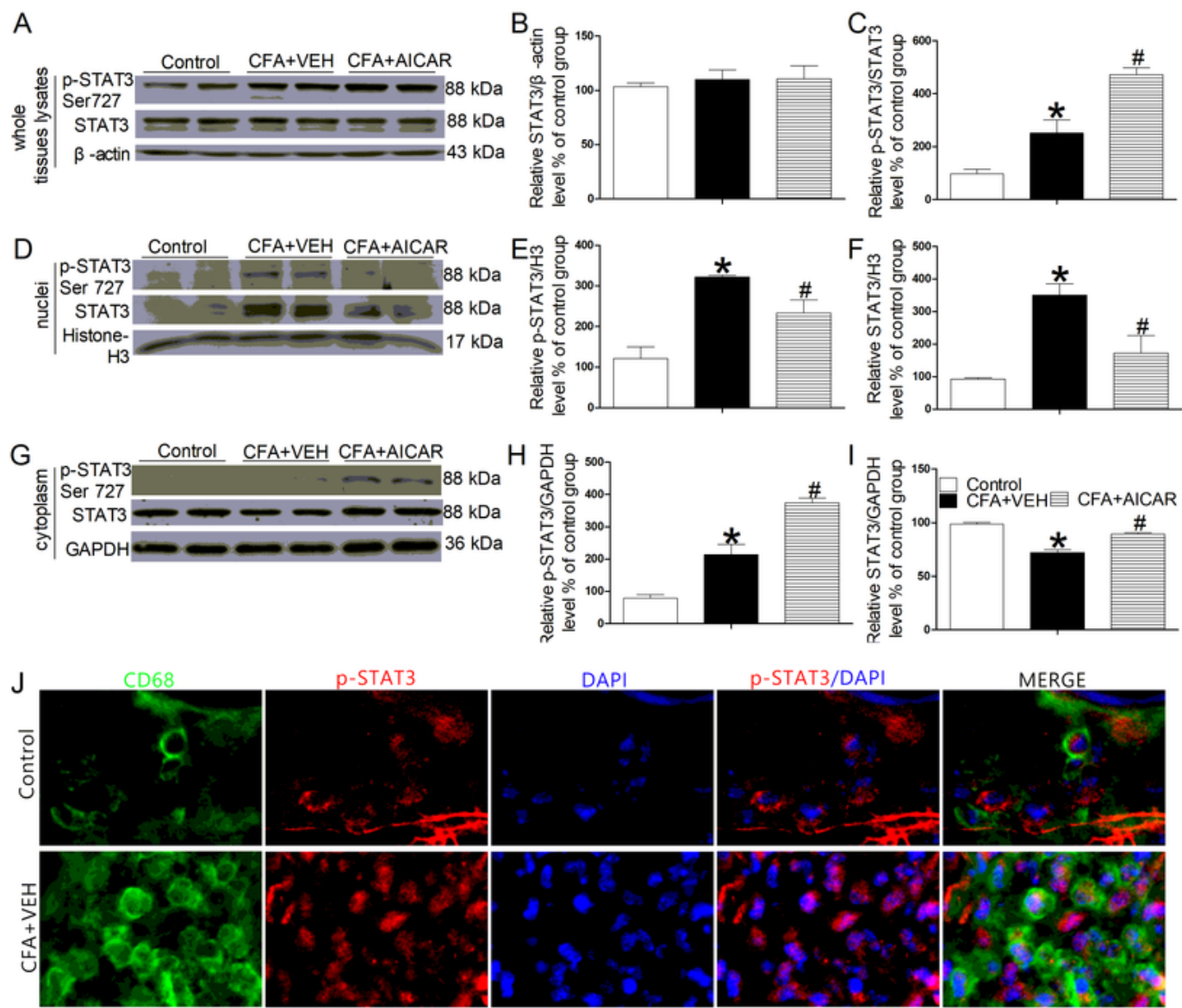

p-STAT3/DAP

MERGE
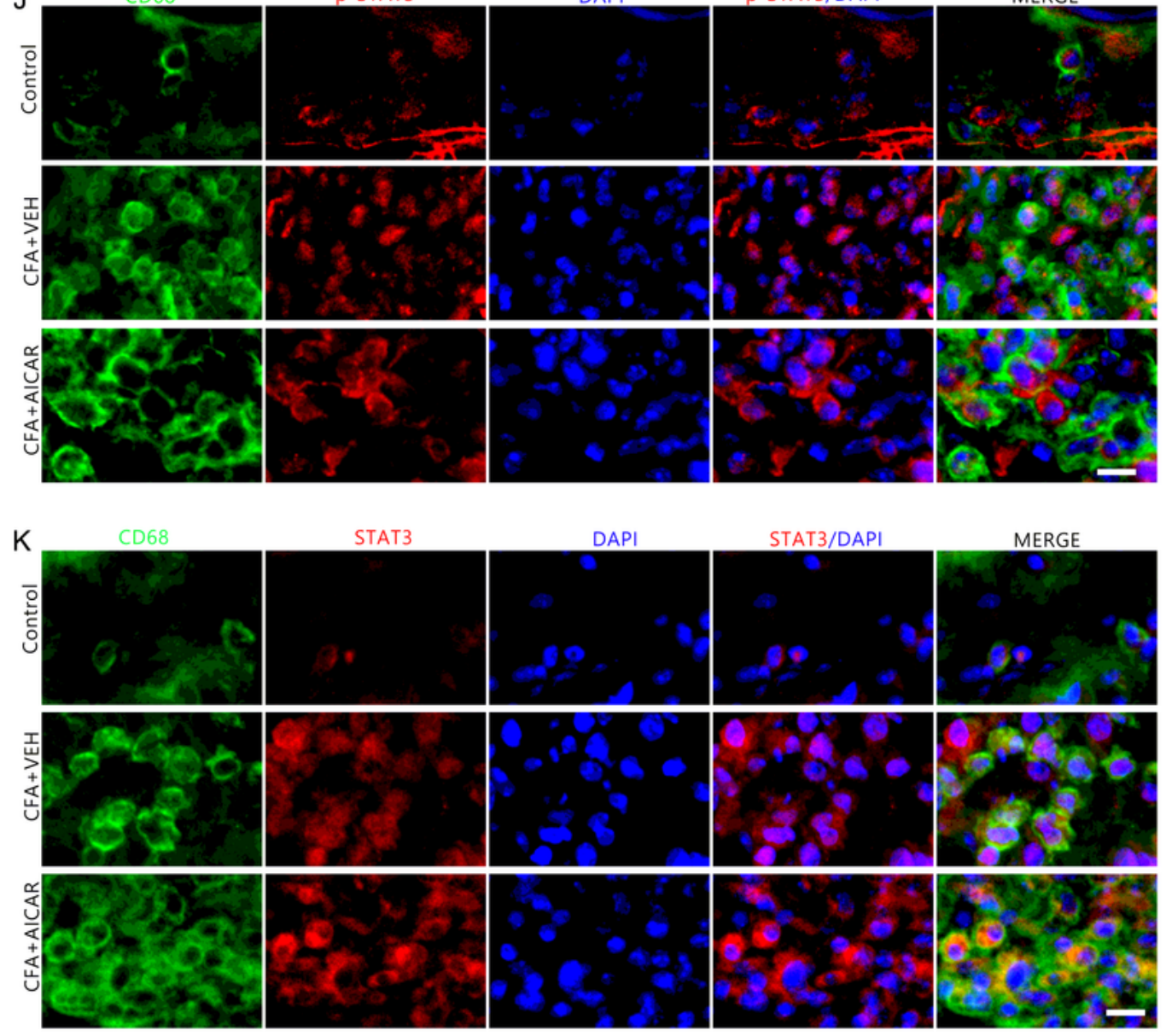

Figure 2

AICAR inhibits STAT3 nuclear translocation and promotes STAT3(Ser727) phosphorylation in the cytoplasm of inflammatory skin tissues. (A, B and C) Gel images and group data of p-STAT3 (Ser727) and STAT3 in total protein of inflamed skin tissues ( $n=3)$. (D, E and F) Gel images and group data of STAT3, p-STAT3 (Ser727) nuclear proteins and a nuclear reference protein Histone-3 (H3) in inflammatory skin tissues(n=3). (G, H and I) Gel images and group data of STAT3, p-STAT3 (Ser727) proteins and an 
internal reference GAPDH in cytoplasm skin tissues $(n=3)$ respectively. AICAR $(20 \mu \mathrm{g} / 20 \mu \mathrm{l})$ was injected locally at day 7 after CFA-induced inflammation. Local inflammatory skin tissues were collected to extract total protein, cytoplasmic and nuclear proteins for blotting 2 hours after AICAR treatment. * $p<0.05$ vs. Control group and $\# p<0.05$ vs. CFA+VEH group (one-way ANOVA). VEH is the vehicle of AICAR $(0.01 \mathrm{M}$ PBS). (J, K)Immunofluorescence images show subcellular distribution and co-localization of p-STAT3 (Ser727) (red), STAT3 (red), macrophage (CD68) (green) and DAPI(blue) in inflamed skin tissues( $\mathrm{n}=3$

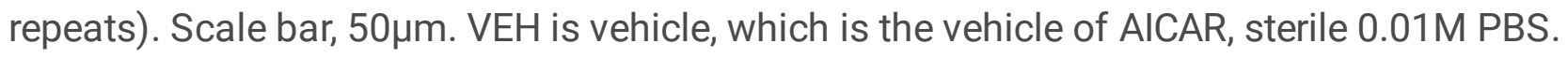

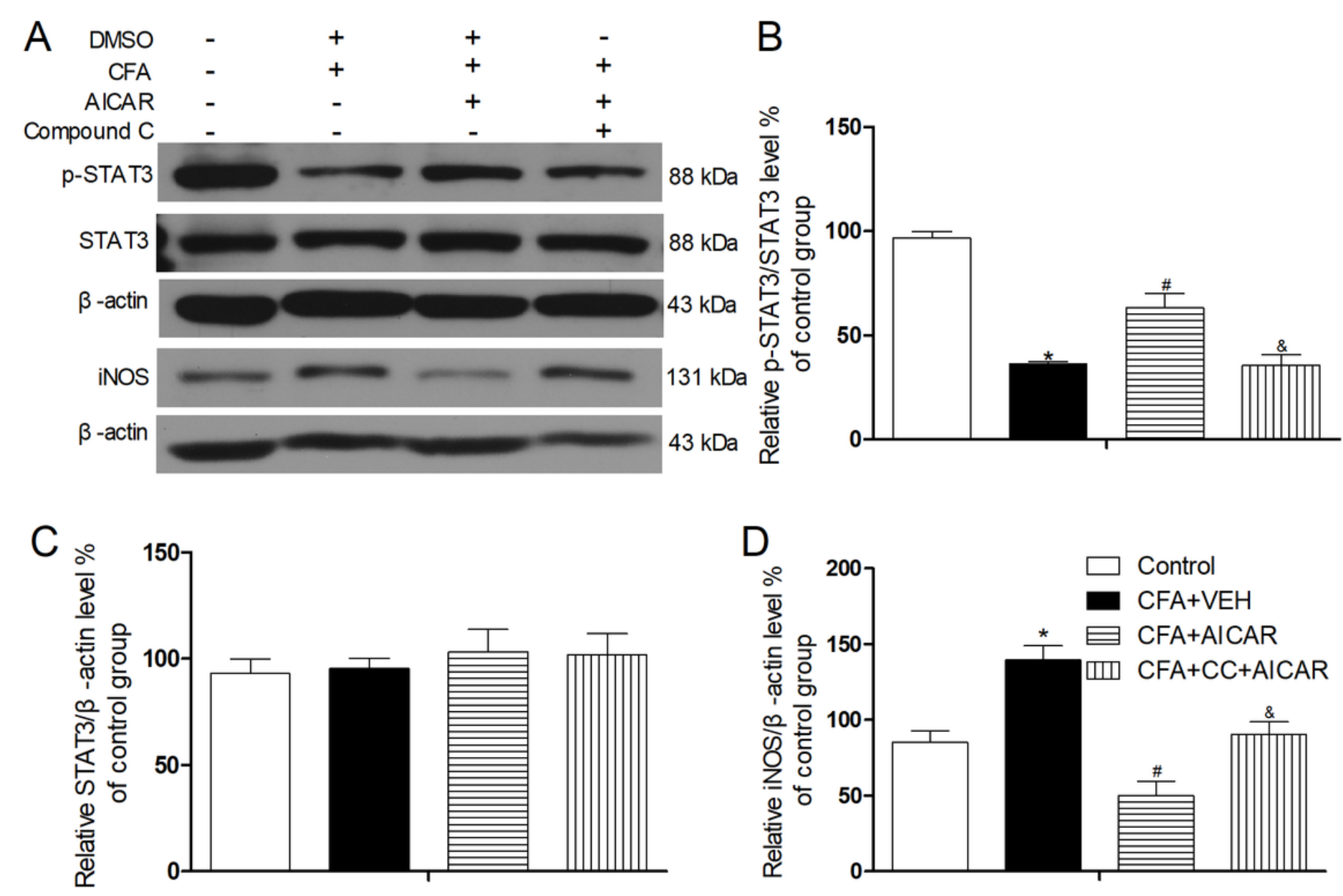

Figure 3

AICAR increases STAT3 (Ser727) phosphorylation and inhibits iNOS expression in NR8383 macrophages treated with CFA. WB was carried out 24 hours after CFA induced inflammatory response. Representative gel images (A) and group data (B, C and D) show p-STAT3(Ser727), STAT3, iNOS and $\beta$-actin in NR8383 cells $24 \mathrm{~h}$ after AICAR treatment ( $n=3$ per group). ${ }^{*} p<0.05$ vs. Control group, $\# p<0.05$ vs. CFA+VEH group and \&p<0.05 vs. CFA+AICAR group (one-way ANOVA). VEH is the vehicle of AICAR (0.01 M PBS). CC, Compound C. 


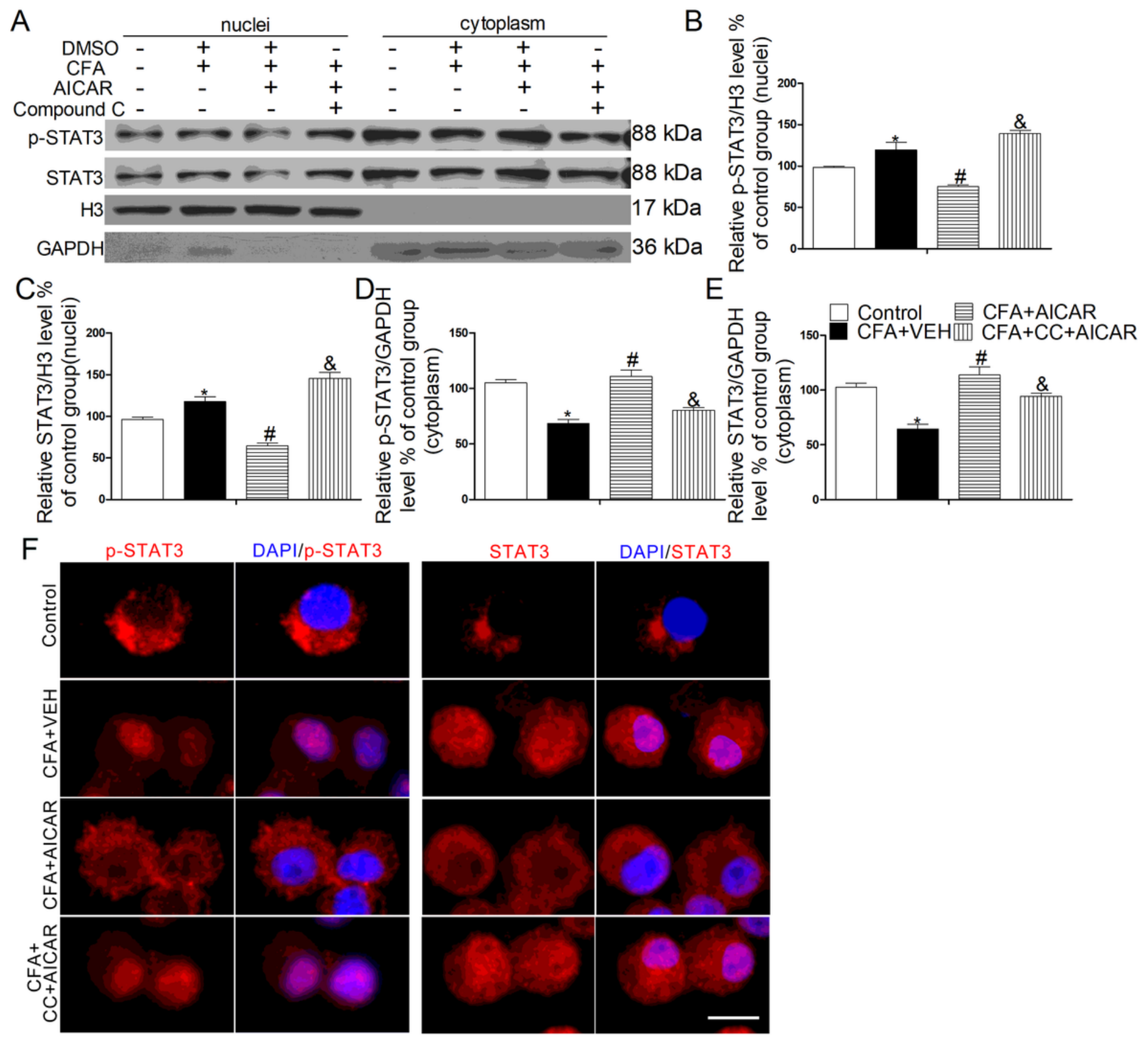

Figure 4

Activation of AMPK inhibits STAT3 nuclear translocation, reduces the level of p-STAT3 (Ser727) in the nuclei, and promotes the phosphorylation of STAT3 (Ser727) in the cytoplasm of NR8383 cells treated with CFA. Cells were collected to extract nuclear and cytoplasmic proteins for immunoblotting 24 hours after AICAR treatment. Gel images (A) of p-STAT3(Ser727), STAT3, Histone-3 and GAPDH proteins in the cytoplasm ( $D$ and $E$ ) and nuclei ( $B$ and $C$ ) of NR8383 ( $n=3$ per group). ${ }^{*} p<0.05$ vs. Control group, $\# p<$ 0.05 vs. CFA+VEH group and \&p<0.05 vs. CFA+AICAR group (One-way ANOVA). VEH is the vehicle of AICAR (0.01 M PBS). CC, Compound C. Co-localization of STAT3 and p-STAT3 (ser727) in macrophages. (F) Immunofluorescence images show the effect of CFA and AICAR on the subcellular distribution of $p$ STAT3 (Ser727) (red), STAT3 (red) and DAPI in NR8383 cells. Scale bar,50 $\mu \mathrm{m}$. 


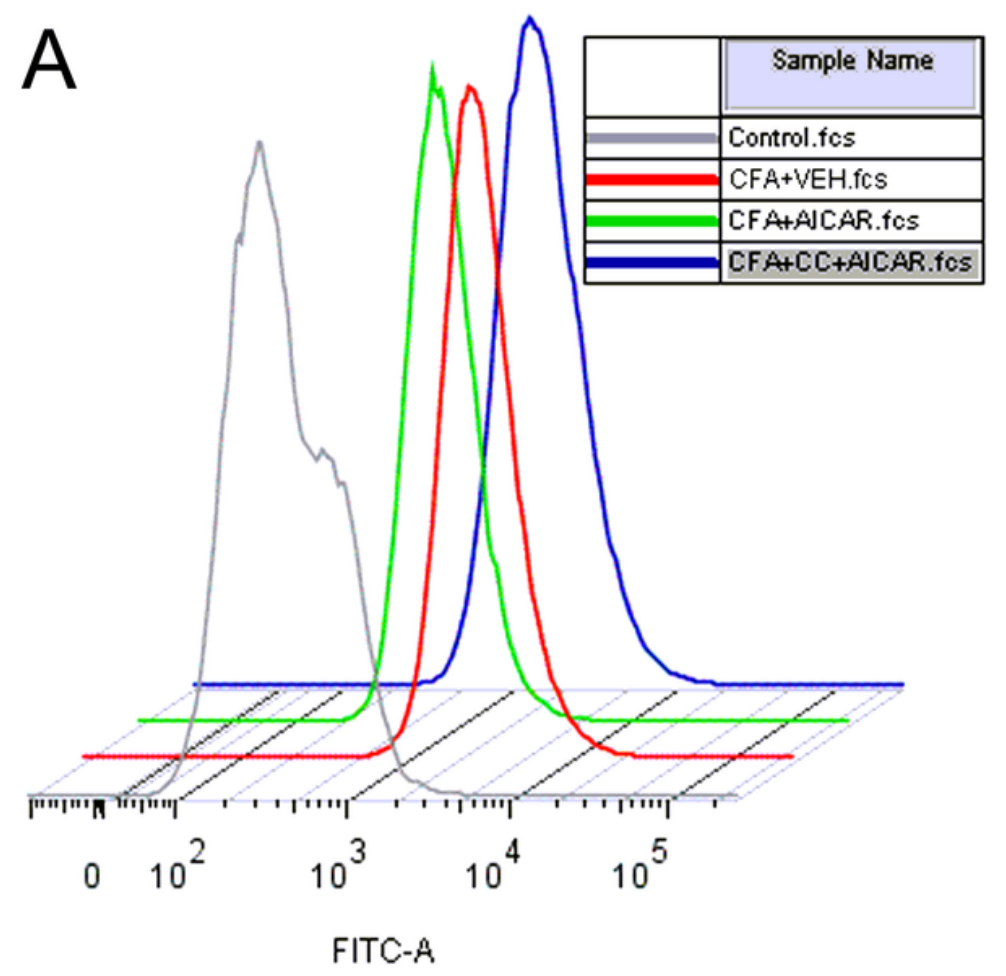

B

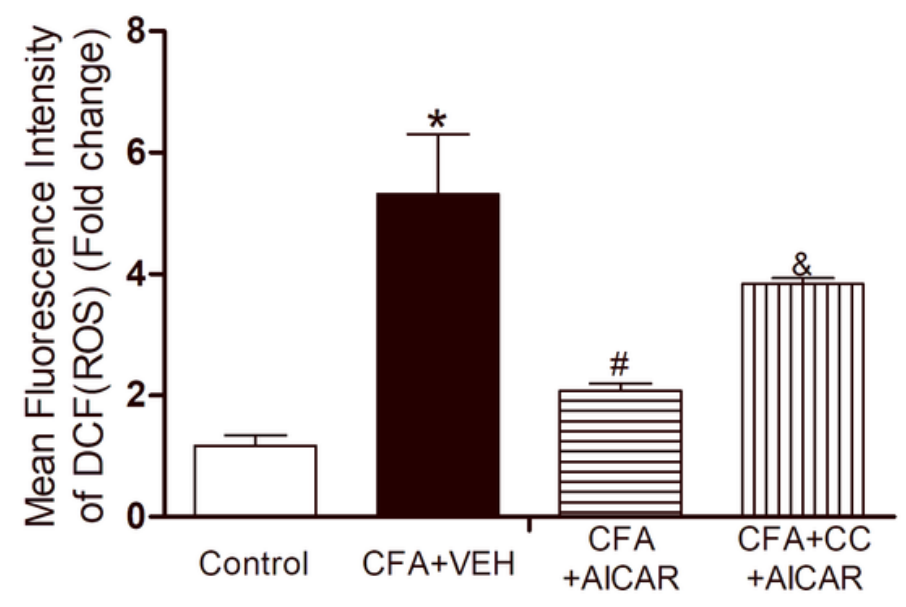

C

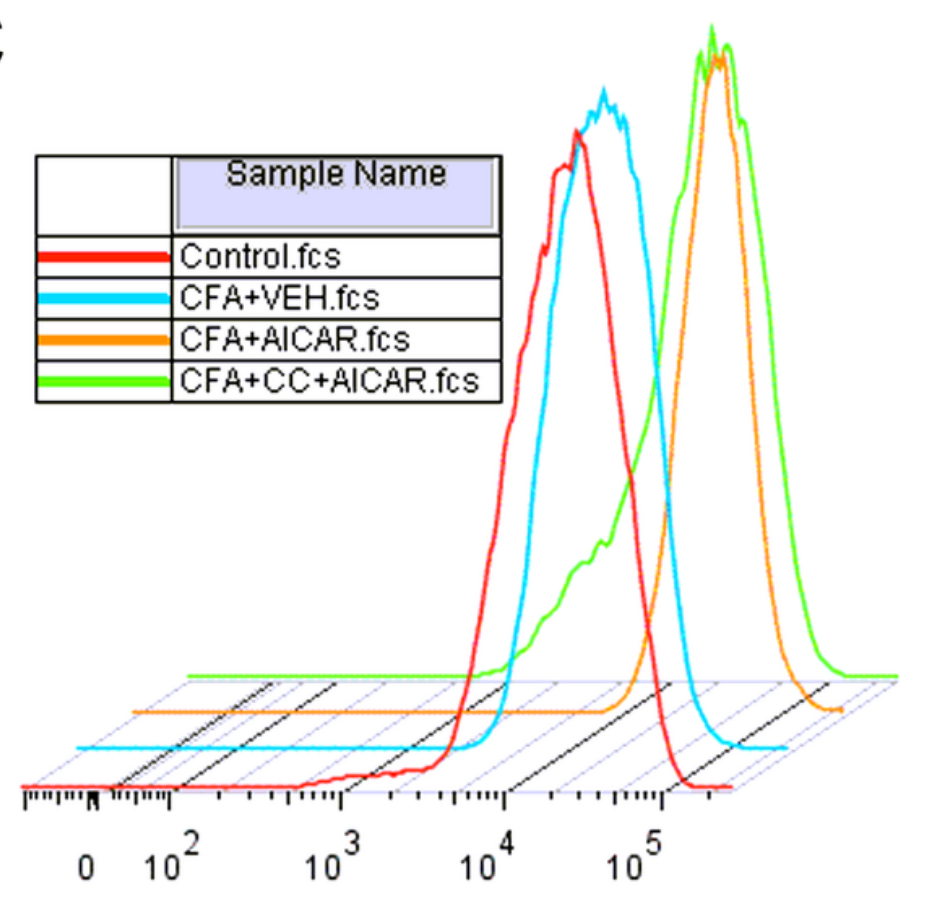

D

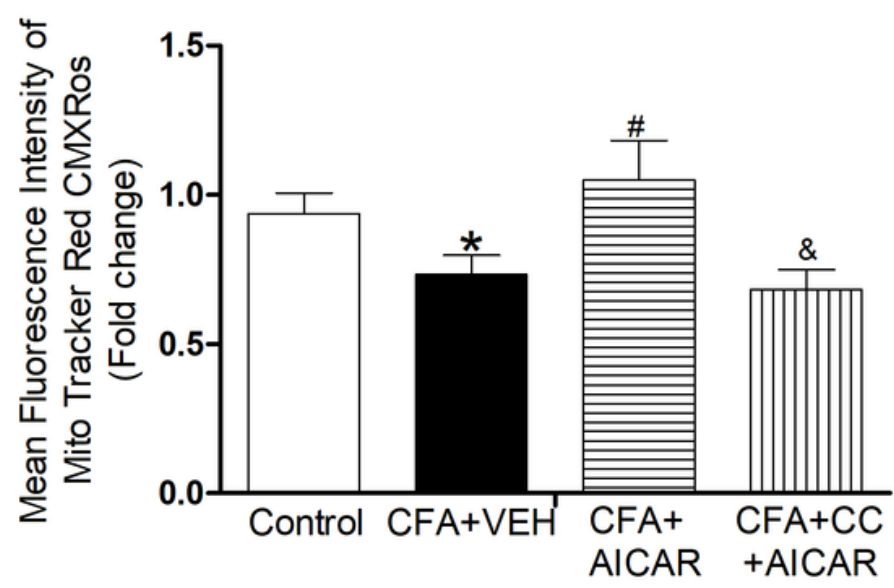

PE-Texas Red-A

Figure 5

Activation of AMPK attenuates ROS accumulation and mitochondrial damage induced by CFA in NR8383 cells. Peak histograms (A) and group data (B) of DCFDA fluorescence intensity ( $n=3$ per group). Peak histograms (C) and group data (D) of fluorescence intensity Mito tracker red CMXRos ( $n=3$ per group). Cells were collected and stained with DCFDA and Mito tracker red CMXRos for flow cytometry 24 hours after AICAR treatment. ${ }^{*} p<0.05$ vs. Control group, $\# p<0.05$ vs. CFA+VEH group and $\& P<0.05$ vs. CFA+AICAR group (one-way ANOVA). VEH is the vehicle of AICAR (0.01 M PBS). CC, Compound C. 
A

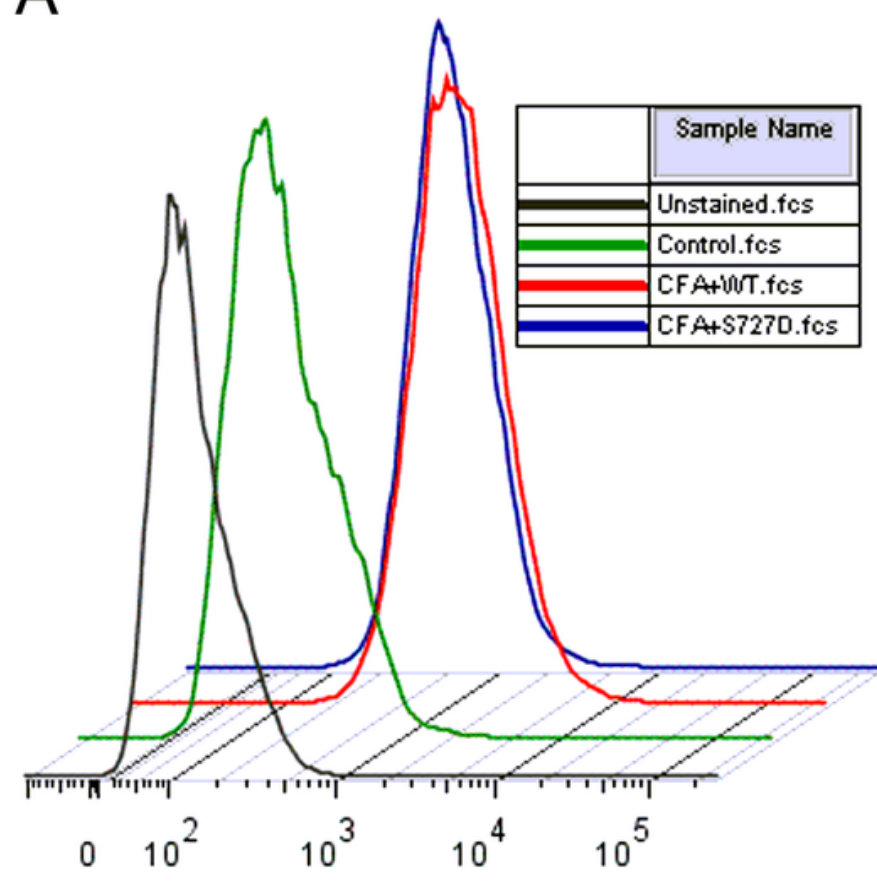

FITC-A

\section{B}

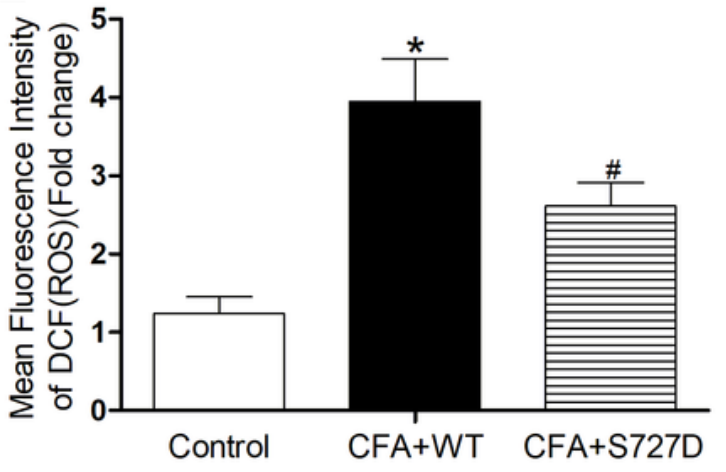

C

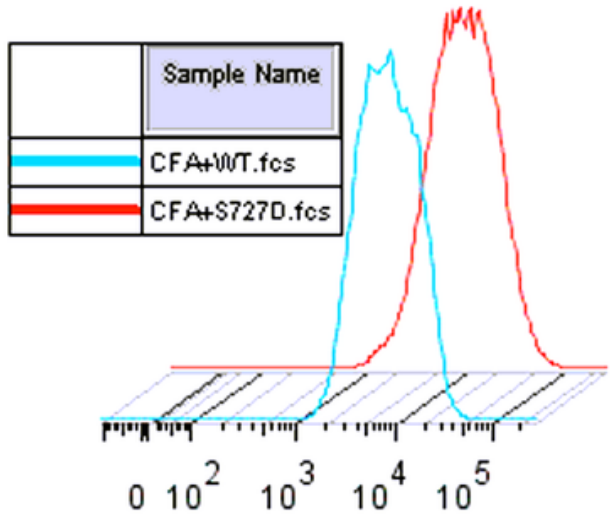

PE-Texas Red-A

D

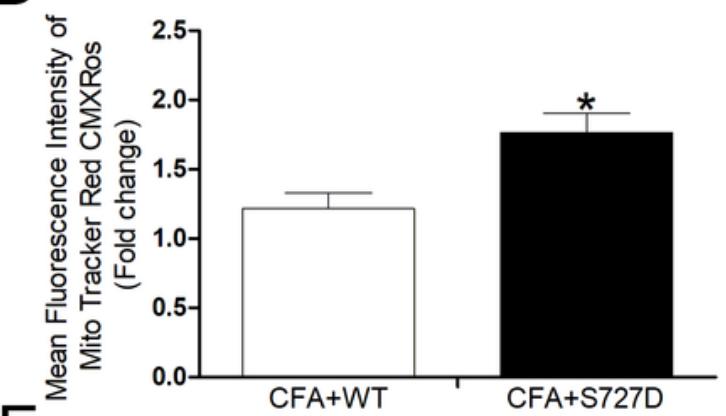

$E^{2}$
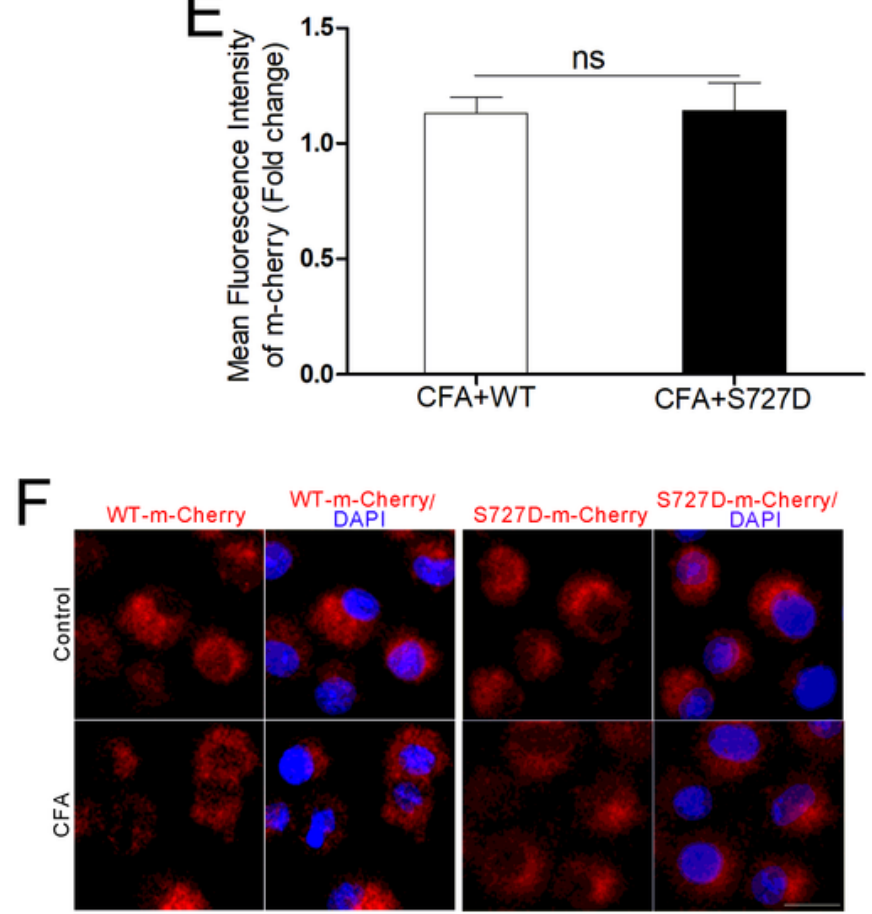

S727D-m-Cherry S727D-m-Cherryl

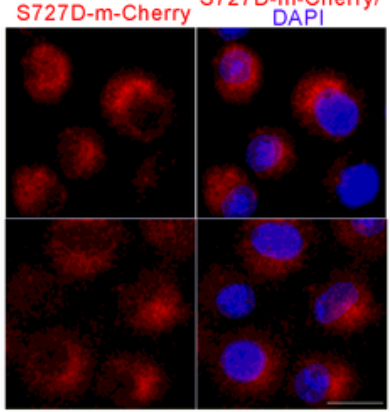

\section{Figure 6}

Promoting STAT3 phosphorylation attenuates ROS accumulation and mitochondrial damage induced by CFA in NR8383 cells. (A, B) Peak histogram and group data of DCF fluorescence intensity ( $n=3$ per group). (C, D) Peak histogram and group data of Mito Tracker Red CMXRos ( $\mathrm{n}=3$ per group) NR8383 cells were transfected with wild-type (m-Cherry-STAT3 WT) or the STAT3 Ser727 mutant (m-Cherry-STAT3 S727D) vectors, and then the cells were treated with CFA $(100 \mu \mathrm{g} / \mathrm{ml})$ for 24 hours before flow cytometry. $\mathrm{k} p<$ 
0.05 vs. Control group and \#p $<0.05$ vs. CFA+WT group (One-way ANOVA in B or unpaired Student's t test in $D$ and $E$ ). ${ }^{*} p<0.05$ vs. Control group ( $n=3$ per group). (E) m-Cherry fluorescence intensity of CFA+WT group and CFA+S727D group without Mito Tracker Red CMXRos staining. (F) Co-labeling image of mCherry and DAPI after 24 hours of N.S. and CFA treatment. The scale bar is $50 \mu \mathrm{m}$.

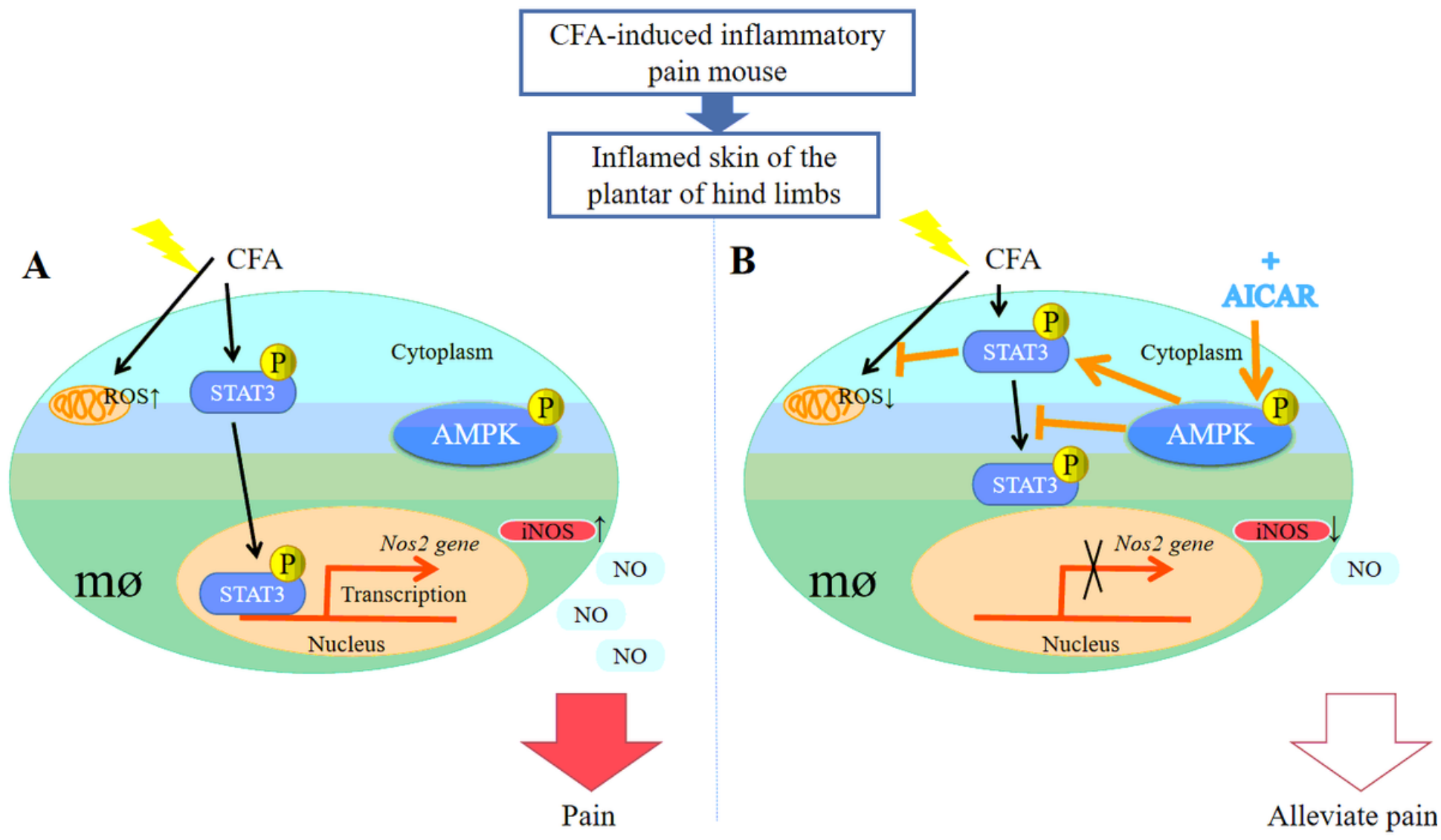

Figure 7

Schematic diagram shows that AMPK activation regulates STAT3 to alleviate inflammatory pain. (A) In CFA-induced inflammatory pain model, CFA causes STAT3 nuclear translocation in macrophages and increases level of p-STAT3 (Ser727) in the nuclei to promote the expression of iNOS. Also, CFA induces ROS accumulation and mitochondrial dysfunction in macrophages. (B) Activating AMPK with AICAR inhibits STAT3 nuclear translocation and decreases p-STAT3 (Ser727) in the nuclei to reduce iNOS in macrophages. Activation of AMPK increases p-STAT3 (Ser727) in the cytoplasm to attenuate CFAinduced mitochondrial damage and ROS accumulation. 